Article

\title{
Crop Sequence Influences on Sustainable Spring Wheat Production in the Northern Great Plains
}

\author{
Donald L. Tanaka *, Mark A. Liebig, Joseph M. Krupinsky and Stephen D. Merrill \\ Agricultural Research Service, United States Department of Agriculture (USDA-ARS), \\ P.O. Box 459, Mandan, ND 58554, USA; E-Mails: mark.liebig@ars.usda.gov (M.A.L.); \\ joe1142k@hotmail.com (J.M.K.); steve.merrill@ars.usda.gov (S.D.M.) \\ * Author to whom correspondence should be addressed; E-Mail: don.tanaka@ars.usda.gov; \\ Tel.: +1-701-667-3063; Fax: +1-701-667-3054.
}

Received: 11 October 2010; in revised form: 22 November 2010 / Accepted: 23 November 2010 / Published: 30 November 2010

\begin{abstract}
Cropping systems in American agriculture are highly successful since World War II, but have become highly specialized, standardized, and simplified to meet the demands of an industrialized food system. Minimal attention has been given to the efficient exploitation of crop diversity and the synergistic and/or antagonistic relationships of crops in crop sequences. Objectives of our research were to determine if previous crop sequences have long-term benefits and/or drawbacks on spring wheat seed yield, seed $\mathrm{N}$ concentration, and seed precipitation-use efficiency in the semiarid northern Great Plains, USA. Research was conducted $6 \mathrm{~km}$ southwest of Mandan, ND using a $10 \times 10$ crop matrix technique as a research tool to evaluate multiple crop sequence effects on spring wheat (triticum aestivum L.) production in 2004 and 2005. Spring wheat production risks can be mitigated when second year crop residue was dry pea (Pisium sativum L.) averaged over all first year crop residues. When compared to spring wheat as second year crop residue in the dry year of 2004, dry pea as the second year residue crop resulted in a 30\% spring wheat seed yield increase. Sustainable cropping systems need to use precipitation efficiently for crop production, especially during below average precipitation years like 2004. Precipitation use efficiency average over all treatments, during the below average precipitation year was $23 \%$ greater than the above average precipitation year of 2005 . Diversifying crops in cropping systems improves production efficiencies and resilience of agricultural systems.
\end{abstract}


Keywords: cropping systems; no-till; crop rotation; dryland agriculture

\section{Introduction}

American agriculture represents a highly productive enterprise, producing bountiful harvest for a growing population. However, closer inspection reveals U.S. agricultural production systems that are highly unstable, easily disrupted by weather, disease, and insects, and relies heavily on foreign trade. Cropping systems since World War II have become highly specialized, standardized, and simplified to meet increasing demands of the industrialized food system [1,2]. Many of these cropping systems are approaching monoculture systems that need to incorporate technological advances, high fossil fuel based inputs, and genetic engineering to remain sustainable. Nature's plant and animal diversity is currently replaced with a small number of cultivated plants and domestic animals [3].

Sustainable agriculture is not a constant state but is in a state of flux at all times; production systems that were sustainable twenty to thirty years ago are not sustainable today [4]. The northern Great Plains is an example of constantly changing agriculture. Early settlers brought their tillage tools and annual cropping systems from the eastern U.S. to the northern Great Plains during the late 1890's and early 1900's. They soon learned that eastern U.S. cultural practices and cropping systems resulted in crop failure during dry periods and were not resilient, which led to the development of the wheatfallow cropping systems to provide short-term production sustainability during dry years. We have since learned that fallow in cropping systems contributes to inefficient use of precipitation [5] and soil resource degradation [6]. Canadian scientists have shown that reducing the frequency of fallow and including legumes in the cropping system improved the overall sustainability of agricultural production in the semiarid northern Great Plains [7].

Cropping systems specializing in one or two crops with little attention to crop diversity could lead to biological and physical soil degradation and ultimately soil chemical degradation [2]. Annual cropping systems, which include diverse crops such as oilseeds, pulses, forbs, and forages, may be economically viable options for producers. Diversifying the plant community in cropping systems also influences the diversity of soil organisms and the soil environment. Enhancing biodiversity in agricultural systems can be a key ecological strategy to bring sustainability to cropping systems [3]. Soil organisms and soil environmental changes resulting from diverse plant communities in cropping systems perpetuate biological synergies and adaptive management techniques inherent to multispecies systems [8,9]. Minimal attention has been given to efficient exploitation of synergism and/or antagonism in cropping systems [10]. Attention to synergistic and/or antagonistic relationships of crops in cropping systems based on crop sequence or cropping patterns is crucial to bring sustainability and resilience to agricultural systems.

Objectives of our research were to determine if previous crop sequences have long-term benefits and/or drawbacks on spring wheat seed yield, seed $\mathrm{N}$ concentration, and seed precipitation-use efficiency in the northern Great Plains. 


\section{Materials and Methods}

The research project was located at the Area IV Soil Conservation District/USDA-ARS Northern Great Plains Research Laboratory Research Farm about $6 \mathrm{~km}$ southwest of Mandan, ND ( $46^{\circ} 46^{\prime} 22^{\prime \prime} \mathrm{N}, 100^{\circ} 57^{\prime} 09^{\prime}$ W). Two sites (6.1 ha each) were chosen about $2 \mathrm{~km}$ apart on a Temvik-Wilton silt loam (fine-silty, mixed, superactive, frigid Typic and Pachic Haplustolls). Prior crops were a 3-year rotation of sunflower (Helianthus annus L.) — spring wheat - spring wheat. During the establishment phase, sunflower was seeded using minimum-till techniques and spring wheat was seeded using no-till techniques. Inoculants, fertilizer rates, and other specific details for crop production are described by Tanaka et al. [11].

Research was initiated in 2002 by no-till seeding 10 crops (buckwheat [Fagopyrum esculentum Moench], canola [Brassica napus L.], chickpea [Cicer arietinum L.], corn [Zea mays L.], dry pea, grain sorghum [Sorghum bicolor L.], lentil [Lens culinaris Medik.], proso millet [Panicum miliaceum L.], sunflower, and spring wheat) in adjacent strips to produce their respective crop residues (first year crop residue). The following year, the same 10 crops were no-till seeded perpendicular to the previous year, creating a 10x10 crop x crop residue matrix with 100 different crop sequences (second year crop residue) [11]. In 2003, a second site of the three-year project was conducted using the same crop sequences as those used at the first site. The three-year crop sequences were run from 2002 to 2004 at site 1 and from 2003 to 2005 at site 2 (Table 1 and Figure 1). Spring wheat was uniformly no-till seeded at Site 1 in 2004 and Site 2 in 2005 after the 100 different crop sequence combinations. Using this crop matrix technique as a research tool allows for evaluation of multiple crop sequence effects in the same experiment under similar weather and soil conditions. Thus, spring wheat was no-till seeded over the crop residue of all crops included in the matrix. Crops were arranged each year using a randomized-complete block experimental design with a strip-block treatment arrangement and four replicates. The smallest experimental unit was 9 by $9 \mathrm{~m}$. All crops, except corn and sunflower, were seeded using a no-till drill (model 750, John Deere, Moline, $\mathrm{IL}^{2}$ ) with a 19-cm row spacing. Corn and sunflower were planted with a no-till row-crop planter in $76 \mathrm{~cm}$ rows.

Table 1. Crops and sites used to evaluate influences of crop sequence on spring wheat production.

\begin{tabular}{cccc}
\hline Year & \multicolumn{1}{c}{ Crop } & $\underline{\text { Site 1 }^{\mathbf{a}}}$ & $\underline{\text { Site 2 }^{\mathbf{a}}}$ \\
1 & Ten Crops $^{\mathrm{b}}$ & 2002 & 2003 \\
2 & Crop matrix, ten crops $^{\mathrm{c}}$ & 2003 & 2004 \\
3 & Spring wheat seeded over crop matrix $^{\mathrm{d}}$ & 2004 & 2005 \\
\hline
\end{tabular}

\footnotetext{
${ }^{\text {a }}$ In 2002, two 6.1 ha sites were chosen to provide two site years.

${ }^{\mathrm{b}}$ Two years were required to establish a crop by crop residue matrix (crop matrix). The first year, ten crops were seeded in strips to provide residue into which ten crops were seeded the second year.

${ }^{\mathrm{c}}$ Two years were required to establish a crop matrix. The second year, ten crops were seeded perpendicular over the crop residue of the first year to form a crop matrix.

${ }^{\mathrm{d}}$ Spring wheat was uniformly seeded over the entire crop matrix in year three.
} 
Figure 1. A crop x crop residue matrix used to evaluate the influences of crop sequences on agronomic and environmental attributes [11] (From Agron. J. 99:904-911 (2007), with permission, copyright American Society of Agronomy). During the first year, 10 crops (numbered 1 through 10) were no-till seeded into a uniform crop residue. During the second year, the same 10 crops were no-till seeded perpendicular over the residue of the previous year's crops. Individual plot numbers are assigned for each experimental unit in the replication.

\begin{tabular}{|c|c|c|c|c|c|c|c|c|c|c|c|}
\hline \multicolumn{11}{|c|}{ Crop X Crop Residue Matrix, 10 crops } & \\
\hline \multicolumn{5}{|c|}{ ONE REPLICATE } & & & & & & \multirow[b]{2}{*}{1} & \\
\hline 109 & 119 & 129 & 139 & 149 & 159 & 169 & 179 & 189 & 199 & & \\
\hline 108 & 118 & 128 & 138 & 148 & 158 & 168 & 178 & 188 & 198 & 2 & \\
\hline 107 & 117 & 127 & 137 & 147 & 157 & 167 & 177 & 187 & 197 & 5 & \\
\hline 106 & 116 & 126 & 136 & 146 & 156 & 166 & 176 & 186 & 196 & 9 & $1^{\text {st }}$ year, \\
\hline 105 & 115 & 125 & 135 & 145 & 155 & 165 & 175 & 185 & 195 & 7 & \\
\hline 104 & 114 & 124 & 134 & 144 & 154 & 164 & 174 & 184 & 194 & 10 & strips \\
\hline 103 & 113 & 123 & 133 & 143 & 153 & 163 & 173 & 183 & 193 & 6 & \\
\hline 102 & 112 & 122 & 132 & 142 & 152 & 162 & 172 & 182 & 192 & 3 & \\
\hline 101 & 111 & 121 & 131 & 141 & 151 & 161 & 171 & 181 & 191 & 4 & \\
\hline 100 & 110 & 120 & 130 & 140 & 150 & 160 & 170 & 180 & 190 & 8 & \\
\hline 5 & 2 & 7 & 1 & 8 & 4 & 6 & 9 & 3 & 10 & & \\
\hline
\end{tabular}

In late April of 2004 and 2005, spring wheat (cv. Amidon) was uniformly no-till seeded over Site 1 (2004) and Site 2 (2005) after the 100 different crop sequence combinations. Spring wheat was seeded at 3.2 million viable seeds $\mathrm{ha}^{-1}$, with $\mathrm{N}$ fertilizer $\left(78 \mathrm{~kg} \mathrm{~N}^{-1}\right.$ as ammonium nitrate) banded between every other row in 38-cm spacing and $\mathrm{P}$ fertilizer $\left(11 \mathrm{~kg} \mathrm{P}^{-1}\right.$ as triple superphosphate) with the seed at planting. Prior to seeding, weed control was accomplished using nonselective herbicide (glyphosate [N-(phosphonylmethyl) glycine] $1.1 \mathrm{~kg}$ a.i. $\mathrm{ha}^{-1}$ ) for no-till. Post emergence weed control consisted of fenoxaprop-p-ethyl ( $\mathrm{d}$ isomer) (70 $\mathrm{g}$ a.i. $\mathrm{ha}^{-1}$ ) and 2, 4-D (dichlorophenoxyacetic acid, $350 \mathrm{~g}$ a.i. $\mathrm{ha}^{-1}$ ) applied between Feeks growth stage 3 to 4 [12]. In mid-August, seed yield was determined by harvesting $11.4 \mathrm{~m}^{2}$ area with a plot combine. Seed $\mathrm{N}$ concentration was determined by sending a subsample to a commercial laboratory using the Dumas method for plant $\mathrm{N}$ analysis [13]. Precipitation use efficiency (PUE), an integrative measure of crop sequence influences on water use by spring wheat, was calculated by determining the quantity of precipitation occurring from the harvest of one 
crop to the harvest of spring wheat divided into the actual spring wheat seed yield of each experimental unit.

$$
\text { [PUE }=\text { spring wheat yield / precipitation (harvest to harvest) }] .
$$

A particular crop sequence determined the precipitation received from the harvest of one crop to the harvest of the following spring wheat crop. Merrill et al. [14] determined crop soil water use and soil water content of second year crops at harvest. Soil water remaining after harvest of second year crops was not part of the PUE calculation and may have influenced this following spring wheat production.

Statistical analysis (F test) indicated a significant year (site) x treatment interaction; therefore, each year (site) was analyzed separately (not shown). Spring wheat seed yield and grain $\mathrm{N}$ concentration were analyzed using the GLM procedure [15]. Statistical comparisons within each evaluation were made with Least Significant Difference (LSD). Precipitation-use efficiency comparisons were made using Dunnett's one-tailed test with continuous spring wheat (spring wheat-spring wheat sequence) as the control. Continuous spring wheat was considered to have the lowest yield potential. All statistical differences were evaluated at a probability level of $\leq 0.05$.

\section{Results and Discussion}

\subsection{Growing Season Weather}

Growing season precipitation for 2004 and 2005 were vastly different (Figure 2). Precipitation during the 2004 growing season was $72 \%$ of the long-term average ( $252 \mathrm{~mm}$ ). Only July was close to the average precipitation of $66 \mathrm{~mm}$. In 2005, growing season precipitation was $129 \%$ of the long-term average. About $40 \%$ of the total growing season precipitation in 2005 was during the month of June.

Average monthly temperatures for 2004 were below average for all months with growing season mean temperature $2.4{ }^{\circ} \mathrm{C}$ below the long-term growing season average of $18.1{ }^{\circ} \mathrm{C}$ (Figure 3). The 2004 growing season was one of the five coolest growing seasons on record. For 2005, the average growing season temperature was $17.8^{\circ} \mathrm{C}$ compared to the long-term average of $18.1^{\circ} \mathrm{C}$.

Figure 2. Monthly growing season precipitation in 2004, 2005, and long-term average (1915-2005) at Mandan, ND.

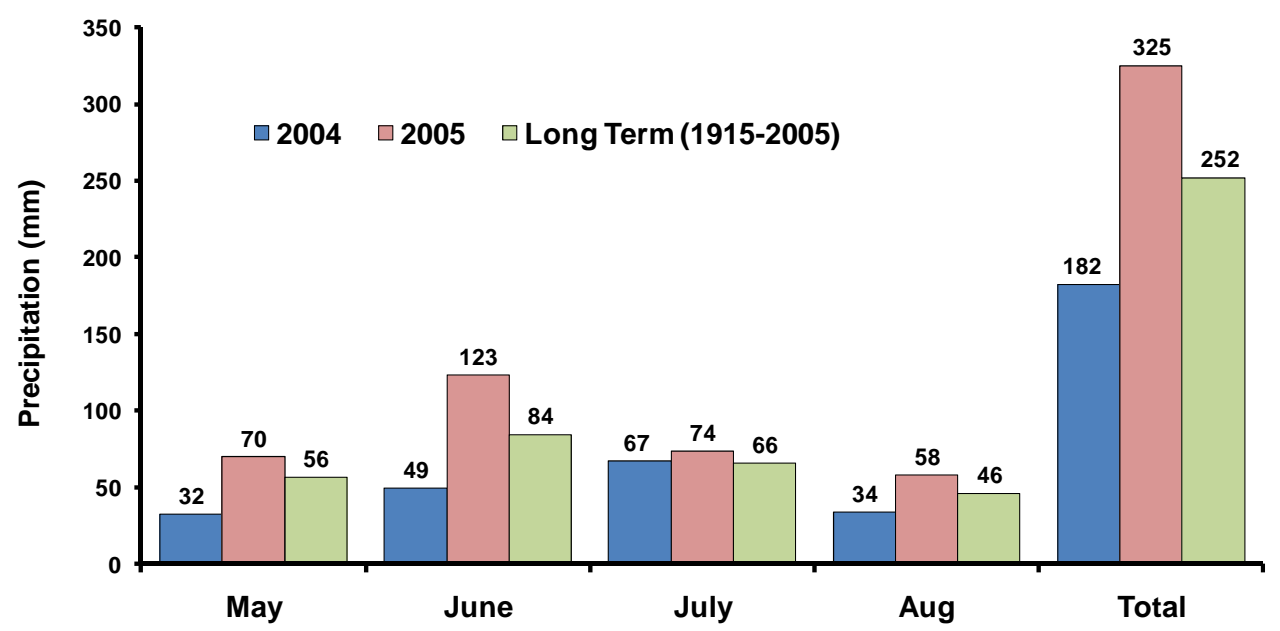


Figure 3. Average monthly growing season temperatures in 2004, 2005, and long-term (1915-2005) at Mandan, ND.

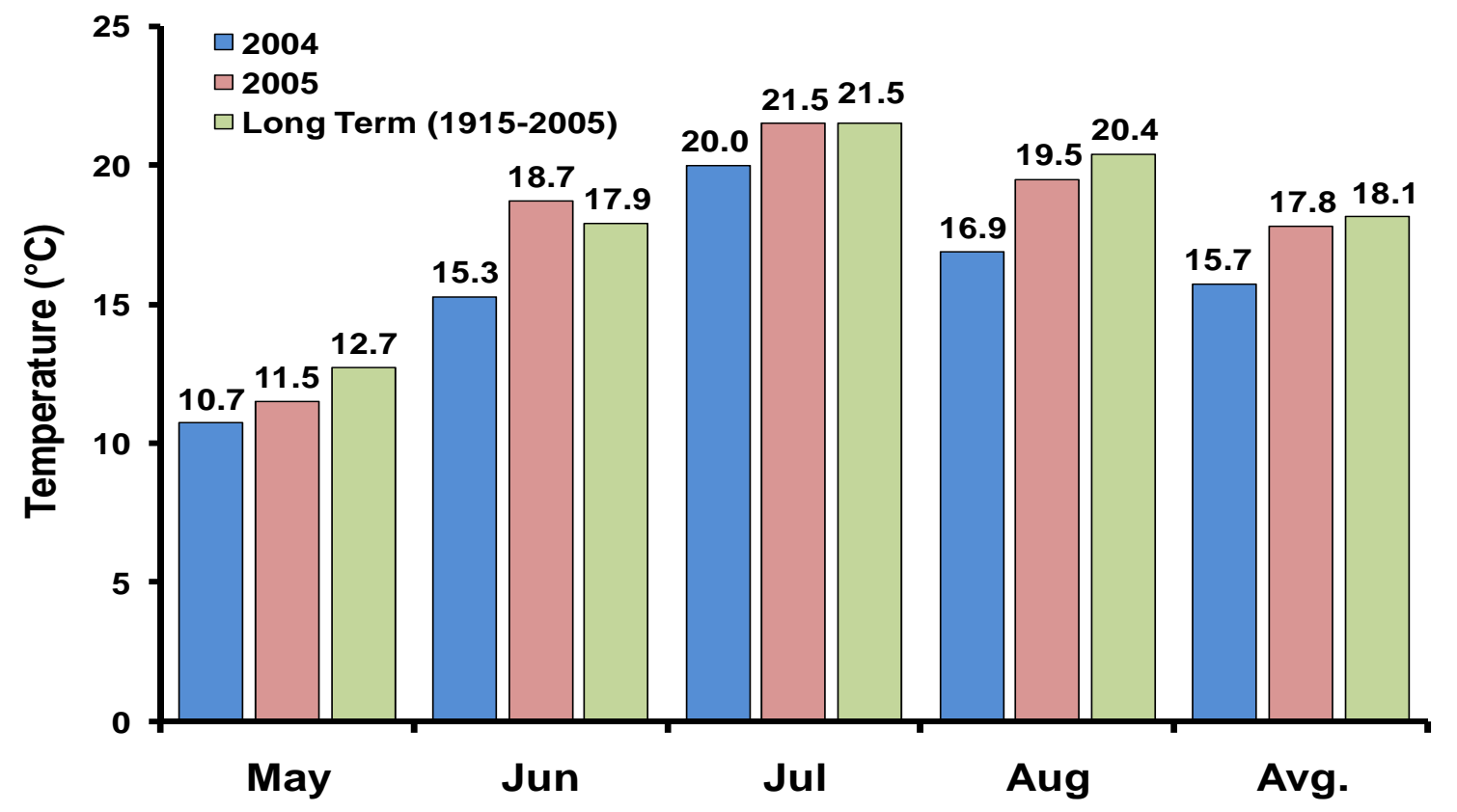

\subsection{Seed Yield}

Spring wheat seed yield for 2004 and 2005 varied among crop sequences, affirming the importance of crop sequence and crop diversity in sustainable spring wheat production for the northern Great Plains (Tables 2 and 3). For both years, when the second year crop residue was dry pea, averaged over all first year crop residues, spring wheat seed yield was the greatest indicating spring wheat production risks can be mitigated by use of crop diversity [16]. In 2004, grain sorghum and sunflower second year crop residues resulted in significantly less spring wheat seed yield when averaged over all first year crop residues (Table 2). Grain sorghum the previous year (second year crop residue) produced significantly greater crop residue than the other ten crops [17] and along with the cooler than average growing season temperature (Figure 3) caused delayed seedling emergence and plant development. The delayed plant development increased plant stress in July when growing season temperatures were about average (Figure 3). When sunflower was grown the previous year (second year crop residue), spring wheat seed yields were $7 \%$ less than the average of spring wheat residue (second year crop residue), because sunflower grown in the northern Great Plains has greater soil water depletion, thus, spring wheat had increased plant stress in July during grain fill [14].

In 2005, growing season conditions were much wetter than 2004 (Figure 2) and crop residue impacts on spring wheat seed yields were not as great as in 2004 (Table 3). When dry pea and millet were the second year crop residue, spring wheat yields were not influenced by first year crop residues. The length of the active growing season for a particular crop greatly influences soil water depletion and succeeding crop growth [18]. Dry pea, and proso millet (second year crop residue) have a short active growing season compared to sunflower or grain sorghum. Therefore, minimal or no differences occur for spring wheat seed yield when grown on short active growing season crop residue, such as dry pea or proso millet, during average or above precipitation years since spring wheat had adequate soil 
water during stress periods. Crops with long active growing seasons (second year crop residue), such as sunflower or grain sorghum, deplete more soil water causing spring wheat stress during the subsequent year. In a crop sequence, first year crops influenced spring wheat seed yield less than second year crops. Therefore, crop sequence and crop choice are critical factors in developing sustainable cropping systems and adaptive management techniques in water-limiting regions such as the northern Great Plains [9].

\subsection{Seed N Concentration}

In 2004, cooler than average growing season temperatures (Figure 3) coupled with below-average precipitation (Figure 2) could have reduced soil and crop residue $\mathrm{N}$ mineralization and resulted in minimal significant differences in spring wheat seed $\mathrm{N}$ concentration among residue combinations (Table 4) [18]. Also, additions of nitrogen fertilizer (78 $\mathrm{kg} \mathrm{N} \mathrm{ha}^{-1}$ ) may have masked any crop sequence influences when $\mathrm{N}$ mineralization from soil and crop residues may have been limited during this below-average precipitation year [18]. Crop sequence combinations of lentil-proso millet and proso millet-lentil residues (first year and second year crop residues, respectively) tended to reduce seed $\mathrm{N}$ concentration. A plausible explanation may be the residue production of proso millet in the lentil-proso millet crop sequence immobilized $\mathrm{N}$ during residue decomposition of the large quantity of high carbon proso millet residue [17]. A similar situation might have occurred for the proso millet-lentil sequence where high carbon proso millet residue from two years prior immobilized $\mathrm{N}$ and resulted in reduced spring wheat seed $\mathrm{N}$ concentration of those no-till crop sequences. A second explanation may be the greater yield of spring wheat from the proso millet-lentil and lentil-proso millet crop sequences lowered $\mathrm{N}$ concentration in the grain through dilution.

In 2005, average growing season temperatures and above-average growing season precipitation (Figures 2 and 3) resulted in spring wheat seed $\mathrm{N}$ concentrations that were lower than 2004 (Table 5). In general, spring wheat seed $\mathrm{N}$ concentration in 2005 was the greatest when canola $\left(29.2 \mathrm{~g} \mathrm{~kg}^{-1}\right)$, buckwheat (29.5 $\left.\mathrm{g} \mathrm{kg}^{-1}\right)$, corn $\left(29.7 \mathrm{~g} \mathrm{~kg}^{-1}\right)$, grain sorghum $\left(29.6 \mathrm{~g} \mathrm{~kg}^{-1}\right)$, proso millet $\left(29.4 \mathrm{~g} \mathrm{~kg}^{-1}\right)$, and spring wheat $\left(29.8 \mathrm{~g} \mathrm{~kg}^{-1}\right)$ were the first year crop residues and least when first year crop residues were chickpea (28.4 $\left.\mathrm{g} \mathrm{kg}^{-1}\right)$, dry pea $\left(28.4 \mathrm{~g} \mathrm{~kg}^{-1}\right)$, and lentil $\left(28.7 \mathrm{~g} \mathrm{~kg}^{-1}\right)$. Spring wheat seed $\mathrm{N}$ concentration for second year crop residue was the greatest for spring wheat $\left(30.7 \mathrm{~g} \mathrm{~kg}^{-1}\right)$ and least for proso millet $\left(27.3 \mathrm{~g} \mathrm{~kg}^{-1}\right)$. No explanation can be given as to why spring wheat seed $\mathrm{N}$ concentration was the highest in spring wheat as second year residue and was the lowest in proso millet as second year residue. Soil N data were not collected, but one can speculate since soil water depletion in 2004 for the crop matrix was greatest for canola, buckwheat, corn, grain sorghum, proso millet and spring wheat crops and least for chickpea, dry pea, and lentil [14]. Greater soil water depletion from crops in 2004 resulted in greater spring wheat grain $\mathrm{N}$ concentration in 2005, with less soil water depletion having lower $\mathrm{N}$ concentration through dilution. 
Table 2. Spring wheat seed yield $\left(\mathrm{kg} \mathrm{ha}^{-1}\right)$ as influenced by first year crop residue and second year crop residue at Mandan, ND in 2004 (Site 1).

\begin{tabular}{|c|c|c|c|c|c|c|c|c|c|c|c|}
\hline \multirow[b]{2}{*}{ First year crop residue (2002) } & \multicolumn{11}{|c|}{ Second year crop residue (2003) } \\
\hline & Buckwheat & Canola & Chickpea & Corn & Dry Pea & Grain Sorghum & Lentil & Proso Millet & Sunflower & Spring Wheat & Average \\
\hline \multirow[t]{2}{*}{ Buckwheat } & $2635 \mathrm{a}^{a}$ & $2107 \mathrm{a}$ & $2314 \mathrm{a}$ & $2391 \mathrm{abc}$ & $2704 \mathrm{bcd}$ & $2218 \mathrm{a}$ & $2660 \mathrm{abcd}$ & $2401 \mathrm{a}$ & $2078 \mathrm{a}$ & $2603 \mathrm{a}$ & \\
\hline & $A^{b}$ & A & A & A & A & A & A & A & A & A & $2411 \mathrm{ZY}^{c}$ \\
\hline \multirow[t]{2}{*}{ Canola } & $2163 \mathrm{a}$ & $2488 \mathrm{a}$ & $2463 \mathrm{a}$ & $1966 \mathrm{~cd}$ & $2825 \mathrm{abc}$ & $1809 a$ & $2692 a b c$ & $2427 \mathrm{a}$ & $2413 \mathrm{a}$ & $2020 \mathrm{a}$ & \\
\hline & A & A & A & A & A & A & A & A & A & A & $2326 \mathrm{ZY}$ \\
\hline \multirow[t]{2}{*}{ Chickpea } & $2362 \mathrm{a}$ & $2479 \mathrm{a}$ & $2300 a$ & $2138 \mathrm{bcd}$ & $2838 \mathrm{abc}$ & $1991 \mathrm{a}$ & $1990 \mathrm{e}$ & $2301 \mathrm{a}$ & $1691 \mathrm{a}$ & $2252 \mathrm{a}$ & \\
\hline & A & A & A & A & A & A & A & A & A & A & $2234 \mathrm{ZYX}$ \\
\hline \multirow[t]{2}{*}{ Corn } & $2061 \mathrm{a}$ & $2309 a$ & $2423 \mathrm{a}$ & $2669 a$ & $2931 \mathrm{abc}$ & $1888 \mathrm{a}$ & 2527 bcde & $2428 \mathrm{a}$ & $2202 \mathrm{a}$ & $2167 \mathrm{a}$ & \\
\hline & A & A & A & A & A & A & A & A & A & A & $2360 \mathrm{ZY}$ \\
\hline \multirow[t]{2}{*}{ Dry Pea } & $2172 \mathrm{a}$ & $2808 \mathrm{a}$ & $2441 \mathrm{a}$ & $2418 \mathrm{ab}$ & $3036 \mathrm{ab}$ & $1859 a$ & 2481 bcde & $2429 a$ & $1923 \mathrm{a}$ & $1972 \mathrm{a}$ & \\
\hline & $\mathrm{CD}$ & $\mathrm{AB}$ & $\mathrm{ABCD}$ & $\mathrm{BCD}$ & A & $\mathrm{D}$ & $\mathrm{ABC}$ & $\mathrm{BCD}$ & $\mathrm{CD}$ & $\mathrm{CD}$ & $2354 \mathrm{ZY}$ \\
\hline \multirow[t]{2}{*}{ Grain Sorghum } & $2088 \mathrm{a}$ & $2108 \mathrm{a}$ & $2568 \mathrm{a}$ & $2361 \mathrm{abc}$ & $2898 \mathrm{abc}$ & $1980 \mathrm{a}$ & $3214 \mathrm{a}$ & $2131 \mathrm{a}$ & $2176 \mathrm{a}$ & $2218 \mathrm{a}$ & \\
\hline & $\mathrm{CD}$ & $\mathrm{CD}$ & $\mathrm{BC}$ & $\mathrm{BCD}$ & $\mathrm{AB}$ & $\mathrm{D}$ & $\mathrm{A}$ & $\mathrm{CD}$ & $\mathrm{CD}$ & $\mathrm{CD}$ & $2374 \mathrm{ZY}$ \\
\hline \multirow[t]{2}{*}{ Lentil } & $2416 \mathrm{a}$ & $2150 \mathrm{a}$ & $2205 \mathrm{a}$ & $2141 \mathrm{bcd}$ & $2402 \mathrm{~cd}$ & $1918 \mathrm{a}$ & $2031 \mathrm{de}$ & $2680 \mathrm{a}$ & $1736 \mathrm{a}$ & $2225 \mathrm{a}$ & \\
\hline & $\mathrm{A}$ & A & $\mathrm{A}$ & $\mathrm{A}$ & A & $\mathrm{A}$ & A & A & A & $\mathrm{A}$ & $2190 \mathrm{YX}$ \\
\hline \multirow[t]{2}{*}{ Proso Millet } & $1917 \mathrm{a}$ & $2456 \mathrm{a}$ & $2520 \mathrm{a}$ & $2723 a$ & $3305 \mathrm{a}$ & $1961 \mathrm{a}$ & $3056 \mathrm{ab}$ & $2679 a$ & $1885 \mathrm{a}$ & $1974 \mathrm{a}$ & \\
\hline & $\mathrm{D}$ & $\mathrm{C}$ & $\mathrm{C}$ & $\mathrm{BC}$ & $\mathrm{A}$ & $\mathrm{D}$ & $\mathrm{AB}$ & $\mathrm{BC}$ & $\mathrm{D}$ & $\mathrm{D}$ & $2447 \mathrm{Z}$ \\
\hline \multirow[t]{2}{*}{ Sunflower } & $2377 \mathrm{a}$ & $2071 \mathrm{a}$ & $2021 \mathrm{a}$ & $1873 d$ & $2121 d$ & $1763 \mathrm{a}$ & $2231 \mathrm{cde}$ & $2222 \mathrm{a}$ & $2134 \mathrm{a}$ & $2117 \mathrm{a}$ & \\
\hline & A & A & A & A & $\mathrm{A}$ & $\mathrm{A}$ & A & A & A & A & $2093 X$ \\
\hline \multirow[t]{2}{*}{ Spring Wheat } & $2229 a$ & $2009 a$ & $2544 \mathrm{a}$ & $2544 \mathrm{ab}$ & $3342 \mathrm{a}$ & $2013 \mathrm{a}$ & $2913 \mathrm{ab}$ & $2308 \mathrm{a}$ & $2014 \mathrm{a}$ & $2245 \mathrm{a}$ & \\
\hline & $\mathrm{C}$ & $\mathrm{C}$ & $\mathrm{BC}$ & $\mathrm{BC}$ & A & $\mathrm{C}$ & $\mathrm{AB}$ & $\mathrm{C}$ & $\mathrm{C}$ & $\mathrm{C}$ & $2416 \mathrm{ZY}$ \\
\hline Average & $2242 \mathrm{xw}^{d}$ & $2298 \mathrm{xw}$ & $2380 \mathrm{yxw}$ & $2322 \mathrm{xw}$ & $2840 \mathrm{z}$ & 1940 u & $2579 \mathrm{y}$ & $2400 \mathrm{yx}$ & $2025 \mathrm{vu}$ & $2179 \mathrm{wv}$ & \\
\hline
\end{tabular}

${ }^{a}$ Small letters in the column that are different indicate significance in spring wheat seed yields as influenced by first year crop residues at $\mathrm{p} \leq 0.05$ according to LSD. ${ }^{b} \mathrm{Capital}$ letters in the row that are different indicate significance in spring wheat seed yields as influenced by second year crop residues at $\mathrm{p} \leq 0.05$ according to LSD. ${ }^{c} \mathrm{Capital}$ letters (first year crop residue in 2002) for spring wheat seed yield averaged across all second year crop residues; average numbers followed by different letters are significant at $\mathrm{p} \leq 0.05$ according to LSD. ${ }^{d}$ Small letters (second year crop residue in 2003) for spring wheat seed yield averaged across all first year crop residues; average numbers followed by different letters and significant at $\mathrm{p} \leq 0.05$ according to LSD. 
Table 3. Spring wheat seed yield $\left(\mathrm{kg} \mathrm{ha}^{-1}\right)$ as influenced by first year crop residue and second year crop residue at Mandan, ND in 2005 (Site 2).

\begin{tabular}{|c|c|c|c|c|c|c|c|c|c|c|c|}
\hline \multirow[b]{2}{*}{ First yr crop residue (2003) } & \multicolumn{10}{|c|}{ Second year crop residue (2004) } & \multirow[b]{2}{*}{ Average } \\
\hline & Buckwheat & Canola & Chickpea & Corn & Dry Pea & Grain Sorghum & Lentil & Proso Millet & Sunflower & Wheat & \\
\hline \multirow[t]{2}{*}{ Buckwheat } & $2785 a b c^{a}$ & $2698 \mathrm{bc}$ & $2751 \mathrm{ab}$ & $2446 a$ & $2963 a$ & $2598 \mathrm{a}$ & $2651 a b c$ & $2605 \mathrm{a}$ & $2675 \mathrm{a}$ & $2296 a b c$ & \\
\hline & $\mathrm{AB}^{b}$ & $\mathrm{ABC}$ & $\mathrm{AB}$ & $\mathrm{CD}$ & A & $\mathrm{BC}$ & $\mathrm{BC}$ & $\mathrm{BC}$ & $\mathrm{BC}$ & $\mathrm{D}$ & $2647 Z^{c}$ \\
\hline \multirow[t]{2}{*}{ Canola } & $2619 \mathrm{~cd}$ & $2736 \mathrm{bc}$ & $2725 \mathrm{ab}$ & $2448 \mathrm{a}$ & $2983 \mathrm{a}$ & $2561 \mathrm{a}$ & $2757 a$ & $2567 \mathrm{a}$ & $2374 \mathrm{bc}$ & $2057 \mathrm{c}$ & \\
\hline & $\mathrm{ABC}$ & $\mathrm{ABC}$ & $\mathrm{ABC}$ & $\mathrm{BC}$ & A & $\mathrm{BC}$ & $\mathrm{AB}$ & $\mathrm{BC}$ & $\mathrm{CD}$ & $\mathrm{D}$ & $2582 \mathrm{ZY}$ \\
\hline \multirow[t]{2}{*}{ Chickpea } & $2866 \mathrm{ab}$ & $2560 \mathrm{c}$ & $2706 \mathrm{abc}$ & $2332 \mathrm{ab}$ & $2808 \mathrm{a}$ & $2096 \mathrm{e}$ & $2463 b c$ & $2417 a$ & $2307 \mathrm{c}$ & $2450 \mathrm{a}$ & \\
\hline & A & $\mathrm{ABC}$ & $\mathrm{AB}$ & $\mathrm{CD}$ & $\mathrm{A}$ & $\mathrm{D}$ & $\mathrm{BC}$ & $\mathrm{BCD}$ & $\mathrm{CD}$ & $\mathrm{BC}$ & $2500 \mathrm{YX}$ \\
\hline \multirow[t]{2}{*}{ Corn } & $2775 a b c$ & $2614 b c$ & $2784 \mathrm{ab}$ & $2409 a$ & $2744 \mathrm{a}$ & $2463 a b c$ & $2679 \mathrm{ab}$ & $2409 a$ & $2388 \mathrm{bc}$ & $2319 \mathrm{ab}$ & \\
\hline & A & $\mathrm{ABCD}$ & $\mathrm{A}$ & $\mathrm{CD}$ & $\mathrm{AB}$ & $\mathrm{BCD}$ & $\mathrm{ABC}$ & $\mathrm{CD}$ & $\mathrm{CD}$ & $\mathrm{D}$ & $2558 \mathrm{ZY}$ \\
\hline \multirow[t]{2}{*}{ Dry Pea } & $2678 \mathrm{bcd}$ & $2693 \mathrm{bc}$ & $2852 \mathrm{a}$ & $2291 \mathrm{ab}$ & $2835 \mathrm{a}$ & 2290 bcde & $2740 a$ & $2546 \mathrm{a}$ & $2451 b c$ & $2291 \mathrm{abc}$ & \\
\hline & $\mathrm{ABC}$ & $\mathrm{ABC}$ & $\mathrm{A}$ & $\mathrm{D}$ & $\mathrm{A}$ & $\mathrm{D}$ & $\mathrm{AB}$ & $\mathrm{BCD}$ & $\mathrm{CD}$ & $\mathrm{D}$ & $2567 \mathrm{ZY}$ \\
\hline \multirow[t]{2}{*}{ Grain Sorghum } & $2807 a b c$ & $2815 \mathrm{ab}$ & $2700 \mathrm{abc}$ & $2433 a$ & $3030 \mathrm{a}$ & $2507 \mathrm{ab}$ & $2873 a$ & $2543 \mathrm{a}$ & $2424 b c$ & $2422 \mathrm{ab}$ & \\
\hline & $\mathrm{AB}$ & $\mathrm{AB}$ & $\mathrm{BC}$ & $\mathrm{D}$ & $\mathrm{A}$ & $\mathrm{CD}$ & $\mathrm{AB}$ & $\mathrm{CD}$ & $\mathrm{D}$ & $\mathrm{D}$ & $2655 \mathrm{Z}$ \\
\hline \multirow[t]{2}{*}{ Lentil } & $2912 \mathrm{a}$ & $2806 \mathrm{ab}$ & $2847 \mathrm{a}$ & $2292 \mathrm{ab}$ & $2968 \mathrm{a}$ & $2197 \mathrm{de}$ & $2764 \mathrm{a}$ & $2732 a$ & $2530 \mathrm{ab}$ & $2499 \mathrm{a}$ & \\
\hline & $\mathrm{A}$ & $\mathrm{ABC}$ & $\mathrm{AB}$ & $\mathrm{D}$ & $\mathrm{A}$ & $\mathrm{D}$ & $\mathrm{ABC}$ & $\mathrm{ABC}$ & $\mathrm{BCD}$ & $\mathrm{CD}$ & $2655 \mathrm{Z}$ \\
\hline \multirow[t]{2}{*}{ Proso Millet } & $2778 \mathrm{abc}$ & $2980 \mathrm{a}$ & $2926 \mathrm{a}$ & $2292 \mathrm{ab}$ & $2962 \mathrm{a}$ & 2254 bcde & $2782 a$ & $2428 \mathrm{a}$ & $2539 \mathrm{ab}$ & $2526 \mathrm{a}$ & \\
\hline & $\mathrm{AB}$ & $\mathrm{A}$ & $\mathrm{A}$ & $\mathrm{C}$ & $\mathrm{A}$ & $\mathrm{C}$ & $\mathrm{AB}$ & $\mathrm{C}$ & $\mathrm{BC}$ & $\mathrm{BC}$ & $2647 \mathrm{Z}$ \\
\hline \multirow[t]{2}{*}{ Sunflower } & $2735 a b c$ & $2549 c$ & $2486 c$ & $2193 b$ & $2793 \mathrm{a}$ & 2234 cde & $2430 c$ & $2571 \mathrm{a}$ & $2330 c$ & $2394 \mathrm{ab}$ & \\
\hline & $\mathrm{AB}$ & $\mathrm{ABC}$ & $\mathrm{BCD}$ & $\mathrm{D}$ & $\mathrm{A}$ & $\mathrm{D}$ & $\mathrm{CD}$ & $\mathrm{ABC}$ & $\mathrm{CD}$ & $\mathrm{CD}$ & 2471 YX \\
\hline \multirow[t]{2}{*}{ Wheat } & $2475 d$ & $2586 \mathrm{c}$ & $2588 \mathrm{bc}$ & $2148 b$ & $2787 \mathrm{a}$ & $2403 \mathrm{abcd}$ & $2485 b c$ & $2272 a$ & $2269 c$ & $2179 b c$ & \\
\hline & $\mathrm{ABCD}$ & $\mathrm{AB}$ & $\mathrm{AB}$ & $\mathrm{D}$ & $\mathrm{A}$ & $\mathrm{BCD}$ & $\mathrm{ABC}$ & $\mathrm{BCD}$ & $\mathrm{BCD}$ & $\mathrm{CD}$ & $2419 X$ \\
\hline Average & $2743 y^{d}$ & $2704 \mathrm{y}$ & $2736 y$ & $2328 \mathrm{v}$ & $2887 \mathrm{z}$ & $2360 \mathrm{wv}$ & $2662 y$ & $2509 x$ & $2429 \mathrm{xw}$ & $2343 \mathrm{wv}$ & \\
\hline
\end{tabular}

${ }^{a}$ Small letters in the column that are different indicate significance in spring wheat seed yields as influenced by first year crop residues at $\mathrm{p} \leq 0.05$ according to LSD. ${ }^{b} \mathrm{Capital}$ letters in the row that are different indicate significance in spring wheat seed yields as influenced by second year crop residues at $\mathrm{p} \leq 0.05$ according to LSD. ${ }^{c} \mathrm{Capital}$ letters (first year crop residue in 2003) for spring wheat seed yield average across all second year crop residues; average numbers followed by different letters are significant at $\mathrm{p} \leq 0.05$ according to LSD. ${ }^{d}$ Small letters (second year crop residue in 2004) for spring wheat seed yield averaged across all first year crop residues; average numbers followed by different letters are significant at $\mathrm{p} \leq 0.05$ according to LSD. 
Table 4. Spring wheat seed $\mathrm{N}$ concentration $\left(\mathrm{g} \mathrm{kg}^{-1}\right)$ as influenced by first year crop residue and second year crop residue at Mandan, ND in 2004 (Site 1).

\begin{tabular}{|c|c|c|c|c|c|c|c|c|c|c|c|}
\hline \multirow[b]{2}{*}{ First yr crop residue (2002) } & \multicolumn{10}{|c|}{ Second year crop residue (2003) } & \multirow[b]{2}{*}{ Average } \\
\hline & Buckwheat & Canola & Chickpea & Corn & Dry Pea & Grain Sorghum & Lentil & Proso Millet & Sunflower & Spring Wheat & \\
\hline \multirow[t]{2}{*}{ Buckwheat } & $33.6 \mathrm{a}^{a}$ & $33.7 \mathrm{a}$ & $34.0 \mathrm{a}$ & $34.0 \mathrm{abc}$ & $32.5 \mathrm{a}$ & $33.9 \mathrm{bcd}$ & $32.8 \mathrm{a}$ & $32.6 \mathrm{a}$ & $33.9 \mathrm{a}$ & $32.5 \mathrm{a}$ & \\
\hline & $\mathrm{A}^{b}$ & $\mathrm{~A}$ & $\mathrm{~A}$ & $\mathrm{~A}$ & $\mathrm{~A}$ & $\mathrm{~A}$ & $\mathrm{~A}$ & $\mathrm{~A}$ & $\mathrm{~A}$ & $\mathrm{~A}$ & $33.3 \mathrm{ZY}^{\mathrm{c}}$ \\
\hline \multirow[t]{2}{*}{ Canola } & $34.1 \mathrm{a}$ & $33.3 \mathrm{a}$ & $33.7 \mathrm{a}$ & 33.4 bcde & $32.6 \mathrm{a}$ & $34.3 \mathrm{ab}$ & $32.9 \mathrm{a}$ & $33.6 \mathrm{a}$ & $33.6 \mathrm{a}$ & $33.6 \mathrm{a}$ & \\
\hline & A & $\mathrm{A}$ & $\mathrm{A}$ & $\mathrm{A}$ & $\mathrm{A}$ & $\mathrm{A}$ & $\mathrm{A}$ & $\mathrm{A}$ & $\mathrm{A}$ & $\mathrm{A}$ & $33.5 \mathrm{Z}$ \\
\hline \multirow[t]{2}{*}{ Chickpea } & $33.7 \mathrm{a}$ & $32.9 \mathrm{a}$ & $33.5 \mathrm{a}$ & $33.9 \mathrm{abcd}$ & $32.7 \mathrm{a}$ & $34.1 \mathrm{abc}$ & $32.9 \mathrm{a}$ & $32.0 \mathrm{a}$ & $34.7 \mathrm{a}$ & $32.7 \mathrm{a}$ & \\
\hline & $\mathrm{A}$ & $\mathrm{A}$ & $\mathrm{A}$ & $\mathrm{A}$ & $\mathrm{A}$ & $\mathrm{A}$ & $\mathrm{A}$ & $\mathrm{A}$ & $\mathrm{A}$ & $\mathrm{A}$ & $33.3 \mathrm{ZY}$ \\
\hline \multirow[t]{2}{*}{ Corn } & $33.6 \mathrm{a}$ & $33.6 \mathrm{a}$ & $32.8 \mathrm{a}$ & $33.0 \mathrm{cdef}$ & $31.9 \mathrm{a}$ & $33.2 \mathrm{~cd}$ & $32.4 \mathrm{a}$ & $32.0 \mathrm{a}$ & $33.2 \mathrm{a}$ & $31.9 \mathrm{a}$ & \\
\hline & $\mathrm{A}$ & $\mathrm{A}$ & $\mathrm{A}$ & $\mathrm{A}$ & $\mathrm{A}$ & $\mathrm{A}$ & $\mathrm{A}$ & $\mathrm{A}$ & $\mathrm{A}$ & $\mathrm{A}$ & $32.8 \mathrm{Y}$ \\
\hline \multirow[t]{2}{*}{ Dry Pea } & $34.8 \mathrm{a}$ & $32.8 \mathrm{a}$ & $33.6 \mathrm{a}$ & 33.6 bcde & $32.8 \mathrm{a}$ & $34.6 \mathrm{ab}$ & $32.6 \mathrm{a}$ & $33.4 \mathrm{a}$ & $34.7 \mathrm{a}$ & $32.6 \mathrm{a}$ & \\
\hline & $\mathrm{A}$ & $\mathrm{A}$ & $\mathrm{A}$ & $\mathrm{A}$ & $\mathrm{A}$ & $\mathrm{A}$ & $\mathrm{A}$ & $\mathrm{A}$ & $\mathrm{A}$ & $\mathrm{A}$ & $33.5 \mathrm{Z}$ \\
\hline \multirow[t]{2}{*}{ Grain Sorghum } & $33.7 \mathrm{a}$ & $33.5 \mathrm{a}$ & $33.2 \mathrm{a}$ & $32.8 \mathrm{ef}$ & $32.6 \mathrm{a}$ & $32.9 \mathrm{~d}$ & $30.7 \mathrm{a}$ & $33.3 \mathrm{a}$ & $33.7 \mathrm{a}$ & $32.3 \mathrm{a}$ & \\
\hline & $\mathrm{A}$ & $\mathrm{A}$ & $\mathrm{A}$ & $\mathrm{A}$ & $\mathrm{A}$ & $\mathrm{A}$ & $\mathrm{A}$ & $\mathrm{A}$ & $\mathrm{A}$ & $\mathrm{A}$ & $32.9 \mathrm{Y}$ \\
\hline \multirow[t]{2}{*}{ Lentil } & $34.6 \mathrm{a}$ & $34.0 \mathrm{a}$ & $33.9 \mathrm{a}$ & $34.3 \mathrm{ab}$ & $33.2 \mathrm{a}$ & $35.0 \mathrm{a}$ & $32.7 \mathrm{a}$ & $32.4 \mathrm{a}$ & $34.5 \mathrm{a}$ & $32.7 \mathrm{a}$ & \\
\hline & $\mathrm{AB}$ & $\mathrm{ABCD}$ & $\mathrm{ABCD}$ & $\mathrm{ABC}$ & $\mathrm{BCD}$ & $\mathrm{A}$ & $\mathrm{CD}$ & $\mathrm{D}$ & $\mathrm{AB}$ & $\mathrm{CD}$ & $33.7 \mathrm{Z}$ \\
\hline \multirow[t]{2}{*}{ Proso Millet } & $34.8 \mathrm{a}$ & $33.3 \mathrm{a}$ & $32.7 \mathrm{a}$ & $32.3 \mathrm{f}$ & $32.1 \mathrm{a}$ & 33.8 bcd & $31.3 \mathrm{a}$ & $32.2 \mathrm{a}$ & $33.6 \mathrm{a}$ & $32.3 \mathrm{a}$ & \\
\hline & A & $\mathrm{ABC}$ & $\mathrm{BCD}$ & $\mathrm{BCD}$ & $\mathrm{CD}$ & $\mathrm{AB}$ & $\mathrm{D}$ & $\mathrm{BCD}$ & $\mathrm{ABC}$ & $\mathrm{BCD}$ & $32.8 \mathrm{Y}$ \\
\hline \multirow[t]{2}{*}{ Sunflower } & $33.7 \mathrm{a}$ & $32.7 \mathrm{a}$ & $34.2 \mathrm{a}$ & $34.8 \mathrm{a}$ & $33.9 \mathrm{a}$ & $34.6 \mathrm{ab}$ & $32.7 \mathrm{a}$ & $33.6 \mathrm{a}$ & $33.5 \mathrm{a}$ & $33.2 \mathrm{a}$ & \\
\hline & $\mathrm{A}$ & $\mathrm{A}$ & $\mathrm{A}$ & $\mathrm{A}$ & $\mathrm{A}$ & $\mathrm{A}$ & $\mathrm{A}$ & $\mathrm{A}$ & $\mathrm{A}$ & $\mathrm{A}$ & $33.7 \mathrm{Z}$ \\
\hline \multirow[t]{2}{*}{ Spring Wheat } & $33.4 \mathrm{a}$ & $33.8 \mathrm{a}$ & $34.0 \mathrm{a}$ & $32.9 \mathrm{def}$ & $32.7 \mathrm{a}$ & $33.8 \mathrm{bcd}$ & $32.6 \mathrm{a}$ & $33.2 \mathrm{a}$ & $34.3 \mathrm{a}$ & $32.7 \mathrm{a}$ & \\
\hline & $\mathrm{A}$ & A & $\mathrm{A}$ & $\mathrm{A}$ & A & A & A & $\mathrm{A}$ & $\mathrm{A}$ & $\mathrm{A}$ & $33.3 \mathrm{ZY}$ \\
\hline Average & $34.0 \mathrm{z}^{d}$ & $33.4 \mathrm{yx}$ & $33.5 \mathrm{zy}$ & $33.5 \mathrm{zy}$ & $32.7 \mathrm{w}$ & $34.0 \mathrm{z}$ & $32.3 \mathrm{w}$ & $32.8 \mathrm{xw}$ & $34.0 \mathrm{z}$ & $32.6 \mathrm{w}$ & \\
\hline
\end{tabular}

${ }^{a}$ Small letters in the column that are different indicate significance in spring wheat seed $\mathrm{N}$ concentration as influenced by first year crop residues at $\mathrm{p} \leq 0.05$ according to LSD. ${ }^{b}$ Capital letters in the row that are different indicate significance in spring wheat seed $\mathrm{N}$ concentration as influenced by second year crop residues at $\mathrm{p} \leq 0.05$ according to LSD. ${ }^{c}$ Capital letters (first year crop residue in 2002) for spring wheat seed $\mathrm{N}$ concentration averaged across all second year crop residues; average numbers followed by different letters are significant at $\mathrm{p} \leq 0.05$ according to LSD. ${ }^{d}$ Small letters (second year crop residue in 2003) for spring wheat seed $\mathrm{N}$ concentration averaged across all first year crop residues; average numbers followed by different letters are significant at $\mathrm{p} \leq 0.05$ according to LSD. 
Table 5. Spring wheat seed N concentration $\left(\mathrm{g} \mathrm{kg}^{-1}\right)$ as influenced by first year crop residue and second year crop residue at Mandan, ND in 2005 (Site 2$)$.

\begin{tabular}{|c|c|c|c|c|c|c|c|c|c|c|c|}
\hline \multirow[b]{2}{*}{ First yr crop residue (2003) } & \multicolumn{10}{|c|}{ Second year crop residue (2004) } & \multirow[b]{2}{*}{ Average } \\
\hline & Buckwheat & Canola & Chickpea & Corn & Dry Pea & Grain Sorghum & Lentil & Proso Millet & Sunflower & Spring Wheat & \\
\hline \multirow[t]{2}{*}{ Buckwheat } & $30.4 a^{a}$ & $28.4 \mathrm{abc}$ & $29.4 \mathrm{bc}$ & $30.9 \mathrm{ab}$ & $29.4 \mathrm{a}$ & $29.3 \mathrm{a}$ & $29.2 \mathrm{bcd}$ & $26.9 \mathrm{~cd}$ & $29.5 \mathrm{a}$ & $31.4 \mathrm{a}$ & \\
\hline & $\mathrm{AB}^{b}$ & $\mathrm{C}$ & $\mathrm{BC}$ & $\mathrm{A}$ & $\mathrm{BC}$ & $\mathrm{BC}$ & $\mathrm{BC}$ & $\mathrm{D}$ & $\mathrm{BC}$ & $\mathrm{A}$ & $29.5 \mathrm{ZY}^{\mathrm{c}}$ \\
\hline \multirow[t]{2}{*}{ Canola } & $30.0 \mathrm{ab}$ & $29.5 \mathrm{a}$ & $29.7 \mathrm{bc}$ & $29.6 \mathrm{~cd}$ & $29.7 \mathrm{a}$ & $27.9 \mathrm{~cd}$ & $29.5 \mathrm{~b}$ & 26.8 cde & $29.0 \mathrm{a}$ & $30.9 \mathrm{ab}$ & \\
\hline & $\mathrm{AB}$ & $\mathrm{B}$ & $\mathrm{B}$ & $\mathrm{B}$ & $\mathrm{B}$ & $\mathrm{CD}$ & $\mathrm{B}$ & $\mathrm{D}$ & $\mathrm{BC}$ & $\mathrm{A}$ & $29.2 \mathrm{ZYX}$ \\
\hline \multirow[t]{2}{*}{ Chickpea } & $28.6 \mathrm{~d}$ & $27.0 \mathrm{~d}$ & $29.0 \mathrm{~cd}$ & $29.2 \mathrm{~cd}$ & $29.5 \mathrm{a}$ & $27.2 \mathrm{~cd}$ & $29.4 \mathrm{bc}$ & $25.9 \mathrm{e}$ & $28.1 \mathrm{a}$ & $29.8 \mathrm{~d}$ & \\
\hline & $\mathrm{BC}$ & $\mathrm{DE}$ & $\mathrm{ABC}$ & $\mathrm{ABC}$ & $\mathrm{AB}$ & $\mathrm{D}$ & $\mathrm{AB}$ & E & $\mathrm{CD}$ & $\mathrm{A}$ & $28.4 \mathrm{~W}$ \\
\hline \multirow[t]{2}{*}{ Corn } & $30.2 \mathrm{ab}$ & $28.5 \mathrm{abc}$ & $29.9 \mathrm{~b}$ & $31.4 \mathrm{a}$ & $29.6 \mathrm{a}$ & $29.3 \mathrm{a}$ & $29.1 \mathrm{bcd}$ & $28.4 \mathrm{a}$ & $29.7 \mathrm{a}$ & $31.2 \mathrm{a}$ & \\
\hline & $\mathrm{AB}$ & $\mathrm{C}$ & $\mathrm{B}$ & $\mathrm{A}$ & $\mathrm{BC}$ & $\mathrm{BC}$ & $\mathrm{BC}$ & $\mathrm{C}$ & $\mathrm{BC}$ & $\mathrm{A}$ & $29.7 \mathrm{Z}$ \\
\hline \multirow[t]{2}{*}{ Dry Pea } & $28.4 \mathrm{~d}$ & $28.0 \mathrm{~cd}$ & $28.0 \mathrm{e}$ & $28.7 d$ & $29.4 \mathrm{a}$ & $27.2 \mathrm{~d}$ & $28.2 \mathrm{~d}$ & $27.1 \mathrm{bc}$ & $28.6 \mathrm{a}$ & $30.1 \mathrm{bcd}$ & \\
\hline & $\mathrm{BCD}$ & $\mathrm{BCD}$ & $\mathrm{BCD}$ & $\mathrm{ABC}$ & $\mathrm{AB}$ & $\mathrm{CD}$ & $\mathrm{BCD}$ & $\mathrm{D}$ & $\mathrm{ABCD}$ & $\mathrm{A}$ & $28.4 \mathrm{~W}$ \\
\hline \multirow[t]{2}{*}{ Grain Sorghum } & $29.6 \mathrm{bc}$ & $29.4 \mathrm{ab}$ & $29.9 \mathrm{~b}$ & $30.3 a b c$ & $30.0 \mathrm{a}$ & $29.5 \mathrm{a}$ & $28.9 \mathrm{bcd}$ & $28.2 \mathrm{a}$ & $29.1 \mathrm{a}$ & $31.0 \mathrm{a}$ & \\
\hline & $\mathrm{BCD}$ & $\mathrm{BCD}$ & $\mathrm{ABCD}$ & $\mathrm{AB}$ & $\mathrm{ABC}$ & $\mathrm{BCD}$ & $\mathrm{DE}$ & E & $\mathrm{CDE}$ & $\mathrm{A}$ & $29.6 \mathrm{ZY}$ \\
\hline \multirow[t]{2}{*}{ Lentil } & $29.0 \mathrm{~cd}$ & $27.3 \mathrm{~cd}$ & $29.9 \mathrm{~b}$ & $29.2 \mathrm{~cd}$ & $29.6 \mathrm{a}$ & $27.1 \mathrm{~d}$ & $29.4 b c$ & $26.0 \mathrm{de}$ & $29.1 \mathrm{a}$ & $30.7 a b c$ & \\
\hline & $\mathrm{B}$ & $\mathrm{C}$ & $\mathrm{AB}$ & $\mathrm{B}$ & $\mathrm{B}$ & $\mathrm{C}$ & $\mathrm{B}$ & $\mathrm{D}$ & $\mathrm{B}$ & $\mathrm{A}$ & 28.7 XW \\
\hline \multirow[t]{2}{*}{ Proso Millet } & $29.9 \mathrm{ab}$ & $29.5 \mathrm{a}$ & $29.9 \mathrm{~b}$ & $30.1 \mathrm{bc}$ & $29.6 \mathrm{a}$ & $28.2 \mathrm{bc}$ & $29.7 \mathrm{ab}$ & $27.7 a b c$ & $29.0 \mathrm{a}$ & $30.8 \mathrm{ab}$ & \\
\hline & $\mathrm{AB}$ & $\mathrm{BC}$ & $\mathrm{AB}$ & $\mathrm{AB}$ & $\mathrm{AB}$ & $\mathrm{CD}$ & $\mathrm{AB}$ & $\mathrm{D}$ & $\mathrm{BCD}$ & $\mathrm{A}$ & $29.4 \mathrm{ZY}$ \\
\hline \multirow[t]{2}{*}{ Sunflower } & $29.7 \mathrm{~b}$ & $28.1 \mathrm{bcd}$ & $28.5 \mathrm{de}$ & $29.5 \mathrm{~cd}$ & $29.7 \mathrm{a}$ & $28.9 \mathrm{ab}$ & $28.4 \mathrm{~cd}$ & $27.9 \mathrm{ab}$ & $29.7 \mathrm{a}$ & $30.9 \mathrm{ab}$ & \\
\hline & $\mathrm{B}$ & $\mathrm{C}$ & $\mathrm{C}$ & $\mathrm{B}$ & $\mathrm{B}$ & $\mathrm{BC}$ & $\mathrm{C}$ & $\mathrm{C}$ & $\mathrm{B}$ & $\mathrm{A}$ & $29.1 \mathrm{YX}$ \\
\hline \multirow[t]{2}{*}{ Wheat } & $30.0 \mathrm{ab}$ & $29.7 \mathrm{a}$ & $30.8 \mathrm{a}$ & $29.9 \mathrm{bc}$ & $30.3 \mathrm{a}$ & $28.9 \mathrm{ab}$ & $30.6 \mathrm{a}$ & $28.4 \mathrm{a}$ & $29.5 \mathrm{a}$ & $30.0 \mathrm{~cd}$ & \\
\hline & $\mathrm{ABC}$ & $\mathrm{ABC}$ & A & $\mathrm{ABC}$ & $\mathrm{AB}$ & $\mathrm{CD}$ & $\mathrm{AB}$ & $\mathrm{D}$ & $\mathrm{BCD}$ & $\mathrm{ABC}$ & $29.8 \mathrm{Z}$ \\
\hline Average & $29.6 \mathrm{yxw}^{d}$ & $28.5 \mathrm{v}$ & $29.5 \mathrm{yxw}$ & $29.9 \mathrm{y}$ & $29.7 \mathrm{yx}$ & $28.3 \mathrm{v}$ & $29.2 \mathrm{xw}$ & $27.3 \mathrm{u}$ & $29.1 \mathrm{w}$ & $30.7 \mathrm{z}$ & \\
\hline
\end{tabular}

${ }^{a}$ Small letters in the column that are different indicate significance in spring wheat seed $\mathrm{N}$ concentration as influenced by first year crop residue at $\mathrm{p} \leq 0.05$ according to LSD. ${ }^{b}$ Capital letters in the row that are different indicate significance in spring wheat seed $\mathrm{N}$ concentration as influenced by second year crop residues at $\mathrm{p} \leq 0.05$ according to LSD. ${ }^{c}$ Capital letters (first year crop residue in 2003) for spring wheat seed $\mathrm{N}$ concentration averaged across all second year crop residues; average numbers followed by different letters are significant at $\mathrm{p} \leq 0.05$ according to LSD. ${ }^{d}$ Small letters (second year crop residue in 2004) for spring wheat seed $\mathrm{N}$ concentration averaged across all first year crop residues; average numbers followed by different letters are significant at $\mathrm{p} \leq 0.05$ according to LSD. 


\subsection{Precipitation Use Efficiency (PUE)}

PUE is the interaction of factors such as crop, soil, and environment to efficiently convert sunlight, nutrients, and water into spring wheat seed. PUE is an agro-ecological parameter allowing for the comparison of production efficiencies among crop sequences. During dry years such as 2004, when growing season precipitation was $72 \%$ of the long-term average, PUE was greater $\left(77.6 \mathrm{~kg} \mathrm{ha}^{-1} \mathrm{~mm}^{-1}\right)$ as compared to $2005\left(62.9 \mathrm{~kg} \mathrm{ha}^{-1} \mathrm{~mm}^{-1}\right)$ when growing season precipitation was $128 \%$ of the long-term. Accordingly, frequency and timing of precipitation relative to plant demand greatly influence PUE.

In 2004, the only crop sequence to have significantly greater PUE than continuous spring wheat $\left(68.9 \mathrm{~kg} \mathrm{ha}^{-1} \mathrm{~mm}^{-1}\right.$ ) was the spring wheat-dry pea sequence (102.6 $\mathrm{kg} \mathrm{ha}^{-1} \mathrm{~mm}^{-1}$ ) (Table 6). During dry weather when spring wheat production is limited by water, adding diversity to a cropping system with dry pea could result in a cropping system that uses precipitation more efficiently; therefore, creating a cropping system more sustainable than continuous spring wheat in dry years.

In an above-average precipitation year like 2005, adding crop diversity significantly improved PUE above that for continuous spring wheat crop sequence (45.5 kg ha $\mathrm{mm}^{-1}$ ) (Table 7). When spring wheat was the second year crop residue, PUE was not statistically different from continuous spring wheat crop sequence. Diversifying crops in cropping systems results in chemical composition differences of root exudates which diversifies soil microbial communities, such as fungi and bacteria, in the rhizosphere synergizing a following spring wheat crop to improve sustainability of the system and increase PUE without adding extra inputs to the system [8]. Unusual crop sequences that cannot be explained include first and second year crop residues of chickpea-canola, chickpea-lentil, sunflower-canola, sunflower-lentil, spring wheat-canola, and spring wheat-lentil, which were not statistically different than continuous spring wheat.

Table 6. Spring wheat precipitation-use efficiency (PUE, $\mathrm{kg} \mathrm{ha}^{-1} \mathrm{~mm}^{-1}$ ) as influenced by crop sequence in 2004 (Site 1). PUE in yellow is significantly greater than continuous wheat (Bold, gray background) which was used as the control in Dunnett's one-tailed test.

\begin{tabular}{|c|c|c|c|c|c|c|c|c|c|c|}
\cline { 2 - 12 } \multicolumn{1}{c|}{} & \multicolumn{9}{c|}{ Second year crop residue (2003) } & Wry \\
\hline $\begin{array}{c}\text { First year } \\
\text { crop residue } \\
\text { (2002) }\end{array}$ & Buckwheat & Canola & Chickpea & Corn & $\begin{array}{c}\text { Dry } \\
\text { Pea }\end{array}$ & $\begin{array}{c}\text { Grain } \\
\text { Sorghum }\end{array}$ & Lentil & $\begin{array}{c}\text { Proso } \\
\text { Millet }\end{array}$ & Sunflower & Wheat \\
\hline Buckwheat & 95.8 & 64.7 & 72.9 & 86.9 & 83.0 & 80.6 & 81.7 & 86.9 & 75.5 & 79.9 \\
\hline Canola & 78.6 & 76.4 & 77.6 & 71.5 & 86.8 & 65.8 & 82.7 & 87.9 & 87.7 & 62.0 \\
\hline Chickpea & 85.8 & 76.1 & 72.4 & 77.7 & 87.1 & 72.4 & 61.1 & 83.3 & 61.5 & 69.2 \\
\hline Corn & 74.9 & 70.9 & 76.3 & 97.0 & 90.0 & 68.6 & 77.6 & 87.9 & 80.1 & 66.6 \\
\hline Dry Pea & 78.9 & 86.2 & 76.9 & 87.9 & 93.2 & 67.6 & 76.2 & 88.0 & 69.9 & 60.6 \\
\hline $\begin{array}{c}\text { Grain } \\
\text { Sorghum }\end{array}$ & 75.9 & 64.7 & 80.9 & 85.8 & 89.0 & 72.0 & 98.7 & 77.2 & 79.1 & 68.1 \\
\hline Lentil & 87.8 & 66.0 & 69.4 & 77.8 & 73.7 & 69.7 & 62.4 & 97.0 & 63.1 & 68.3 \\
\hline Proso Millet & 69.7 & 75.4 & 79.4 & 99.0 & 101.5 & 71.3 & 93.8 & 97.0 & 68.5 & 60.6 \\
\hline Sunflower & 86.4 & 63.6 & 63.6 & 68.1 & 65.1 & 64.1 & 68.5 & 80.5 & 77.6 & 65.0 \\
\hline Wheat & 81.0 & 61.7 & 80.1 & 92.5 & 102.6 & 73.2 & 89.5 & 83.6 & 73.2 & $\mathbf{6 8 . 9}$ \\
\hline
\end{tabular}


Table 7. Spring wheat precipitation-use efficiency (PUE, $\mathrm{kg} \mathrm{ha}^{-1} \mathrm{~mm}^{-1}$ ) as influenced by crop sequence in 2005 (Site 2). PUE in yellow are significantly greater than continuous wheat (Bold, gray background) which was used as the control in Dunnett's one-tailed test.

\begin{tabular}{|c|c|c|c|c|c|c|c|c|c|c|}
\cline { 2 - 13 } \multicolumn{1}{c|}{} & \multicolumn{9}{c|}{ Second year crop residue (2004) } \\
\hline $\begin{array}{c}\text { First year } \\
\text { crop residue } \\
(2003)\end{array}$ & Buckwheat & Canola & Chickpea & Corn & $\begin{array}{c}\text { Dry } \\
\text { Pea }\end{array}$ & $\begin{array}{c}\text { Grain } \\
\text { Sorghum }\end{array}$ & Lentil & $\begin{array}{c}\text { Proso } \\
\text { Millet }\end{array}$ & Sunflower & Wheat \\
\hline Buckwheat & 69.2 & 59.2 & 64.6 & 70.9 & 61.9 & 75.3 & 58.2 & 66.0 & 77.6 & 47.9 \\
\hline Canola & 65.1 & 60.0 & 64.0 & 71.0 & 62.3 & 74.2 & 60.5 & 65.1 & 68.8 & 42.9 \\
\hline Chickpea & 71.2 & 56.2 & 63.6 & 67.6 & 58.6 & 60.8 & 54.1 & 61.3 & 66.9 & 51.1 \\
\hline Corn & 69.0 & 57.4 & 65.4 & 69.8 & 57.3 & 71.4 & 58.8 & 61.1 & 69.2 & 48.4 \\
\hline Dry Pea & 66.6 & 59.1 & 67.0 & 66.4 & 59.2 & 66.4 & 60.1 & 64.5 & 71.1 & 47.8 \\
\hline $\begin{array}{c}\text { Grain } \\
\text { Sorghum }\end{array}$ & 69.8 & 61.8 & 63.5 & 70.5 & 63.2 & 72.7 & 63.1 & 64.5 & 70.3 & 50.6 \\
\hline Lentil & 72.4 & 61.6 & 66.9 & 66.4 & 62.0 & 63.7 & 60.6 & 69.3 & 73.4 & 52.2 \\
\hline Proso Millet & 69.0 & 65.4 & 68.8 & 66.4 & 61.8 & 65.3 & 61.1 & 61.6 & 73.6 & 52.7 \\
\hline Sunflower & 68.0 & 55.9 & 58.4 & 63.6 & 58.3 & 64.8 & 53.3 & 65.2 & 67.5 & 50.0 \\
\hline Wheat & 61.5 & 56.8 & 60.8 & 62.3 & 58.2 & 69.7 & 54.5 & 57.6 & 65.8 & 45.5 \\
\hline
\end{tabular}

\section{Summary}

Cropping systems have evolved over the decades into highly specialized production systems that are heavily dependent on fossil fuel inputs and advanced technology. To be sustainable in the future, agriculture needs to move beyond fossil fuel based systems and rely more on renewable systems that are resilient. Research in cropping systems that evaluates crop sequence to improve crop production beyond fossil fuel derived increases in yield may be the first step toward more sustainable agricultural systems. Crop sequence plays a pivotal role in diversifying cropping systems to synergize agro-ecological parameters resulting in improved production efficiencies. In a crop sequence, second year crops influence spring wheat seed yield more than first year crops. Including crops other than spring wheat in a crop sequence synergizes a spring wheat crop improving capture of sunlight, enhancing nutrient uptake, and improving use of precipitation. Enhancing crop biodiversity in cropping systems is a key agro-ecological strategy to develop more sustainable agricultural systems for the future.

\section{Acknowledgements}

We thank R. Kolberg, J. Hartel, D. Schlenker, D. Wetch, M. Hatzenbuhler, J. Gross, and H. Johnson for their assistance with field research, sample collection and processing, statistical analysis, and data summarization. We also thank the Area IV Soil Conservation Districts, Sclerotinia Research Initiative, National Sunflower Association, and New and Emerging Crops for their support of this research. Inclusion of branded product information is for the benefit of the reader and does not imply preference or endorsement by the USDA-Agricultural Research Service. 


\section{References}

1. Brummer, E.C. Diversity, stability, and sustainable American agriculture. Agron. J. 1998, 90, 1-2.

2. Kirschenmann, F. Why American agriculture is not sustainable. Renewable Resour. J. 2002, 20, 6-11.

3. Altieri, M.A. The ecological role of biodiversity in agroecosystems. Agric. Ecosyst. Envron. 1999, 74, 19-31.

4. Hildebrand, P.E. Agronomy's role in sustainable agriculture: Integrated farming systems. J. Prod. Agric. 1990, 3, 285-288.

5. Farahani, H.J.; Peterson, G.A.; Westfall, D.G. Dryland cropping intensification: A fundamental solution to efficient use of precipitation. Adv. Agron. 1998, 64, 97-223.

6. Liebig, M.A.; Tanaka, D.L.; Krupinsky, J.M.; Merrill, S.D.; Hanson, J.D. Dynamic cropping systems: Contributions to improve agroecosystem sustainability. Agron. J. 2007, 99, 899-903.

7. Zentner, R.P.; Campbell, C.A.; Biederbeck, V.O.; Miller, P.R.; Selles, F.; Fernandez, M.R. In search of a sustainable cropping system for the semiarid Canadian prairies. J. Sust. Agric. 2001, 18, 117-136.

8. Kennedy, A.C. Soil microbial diversity in agricultural systems. In Exploring the Role of Diversity in Sustainable Agriculture; Olson, R., Francis, C., Kaffka, S., Eds.; American Society of Agronomy (ASA): Madison, WI, USA, 1995; pp. 35-54.

9. Kirschenmann, F.L. Potential for a new generation of biodiversity in agroecosystems of the future. Agron. J. 2007, 99, 373-376.

10. Francis, C.A. Potential of multiple cropping systems. In Agroecology and Small Farm Development; Altieri, M.A., Hecht, S.B., Eds.; CRC Press: Boca Raton, FL, USA, 1986; pp. 137-150.

11. Tanaka, D.L.; Krupinsky, J.M.; Merrill, S.D.; Liebig, M.A.; Hanson, J.D. Dynamic cropping systems for sustainable crop production in the northern Great Plains. Agron. J. 2007, 99, 904-911.

12. Large, E.C. Growth stages in cereals; illustration of the Feeks scale. Plant Pathol. 1954, 3, 128-129.

13. Padmore, J.M. Protein (Crude) in Animal Feed-Dumas Method, Method No 968.06. In Official Methods of Analysis of the Association of Official Analytical Chemists, 15th ed.; Herlich, K., Ed.; Association of Official Analytical Chemists (AOAC): Arlington, VA, USA, 1990.

14. Merrill, S.D.; Tanaka, D.L.; Krupinsky, J.M.; Liebig, M.A.; Hanson, J.D. Soil water depletion and recharge under ten crop species and applications to the principles of dynamic cropping systems. Agron. J. 2007, 99, 931-938.

15. Littell, R.C.; Milliken, G.A.; Stroup, W.W.; Wolfinger, R.D. SAS System for Mixed Models; SAS Institute: Cary, NC, USA, 1996.

16. Miller, P.R.; Holmes, J.R. Cropping sequence effects of four broadleaf crops on four cereal crops in the northern Great Plains. Agron. J. 2005, 95, 972-979.

17. Krupinsky, J.M.; Merrill, S.D.; Tanaka, D.L.; Liebig, M.A.; Lares, M.T.; Hanson, J.D. Crop residue coverage of soil influenced by crop sequence in no-till system. Agron. J. 2007, 99, 921-930. 
18. Lupwayi, N.Z.; Soon, Y.K. Nitrogen release from field pea residues and soil inorganic $\mathrm{N}$ in a pea-wheat crop rotation in northwestern Canada. Can. J. Plant Sci. 2009, 89, 239-246.

(C) 2010 by the authors; licensee MDPI, Basel, Switzerland. This article is an open access article distributed under the terms and conditions of the Creative Commons Attribution license (http://creativecommons.org/licenses/by/3.0/). 